[19] KieferLe. Congrès International de Laiterie, Berlin, 1937.

[20] Hartmann, Molkerei Zeitung, 1936, p. 1108.

[21] Teichert. Molkerei Zeitung, 1935, p. 1979.

[22] Beli, Peter, Johnson. Journal of Dairy Science, 1928, p. 163-174.

[23] BELl. Chimie et Industrie, 1928, p. 149.

[24] Leviton. Congrès de Printemps de l'American Chemical Society, avril 1939. Le Lait, 1939, p. 693.

[25] Weis Berg. Food Industry, 1934, p. 368.

[26] R. Uhlen. L'Union Laitière, 15 février, 1943.

[27] Kieferle. 11e Congrès International de Laiterie (2e Section), Berlin, 1937.

\title{
LA SIGNIFICATION DE LA RÉACTION DE STORCH, DE LA VITESSE DE MONTÉE DE LA CRÊME AINSI QUE DE LA DÉTERMINATION DES PHOSPHATASES POUR LE CONTROLE DE LA PASTEURISATION DU LAIT
}

\author{
par \\ A. K. VAN BEVER et J. STRAUB \\ Laboratoire du Service d'Inspection des denréus alimentaires à Amsterdam \\ (Fin.)

\section{II. - LA RELATION ENTRE LA CONCENTRATION DES ENZYMES ET BACTÉRIES ET LA TEMPÉRATURE DE PASTEURISATION}

\section{A. Déduction théorique}

VAN ECK (23) a recherché à quel pourcentage de la valeur originale la teneur en peroxydase descendait, quand on chauffait le lait à une certaine température pendant des durées différentes. Il effectuait ses mesures en comparant chaque fois la coloration bleue, obtenue au moyen de la réaction de Storch, à une échelle, élaborée en effectuant la réaction de Storch dans des mélanges de lait pasteurisé et de lait cru avec proportion ascendante de ce dernier. La destruction de l'enzyme semblait se passer selon une réaction du premier ordre. ZILva (24) s'est livré à la même recherche ; il déterminait ensuite $K$ de la même façon pour toute une série de températures. Bouma et VAN DAM (25) ont montré que les valeurs de Van Eck pour $K$ concordent parfaitement avec les expériences de ZiLvA.

(23) J. J. vaN Eor. Z. Untersuch. Nahr. Genussm., 22, 393, 1911.

(24) S S. ZilvA. Bioch. Journ., 8, 656, 1914.

(25) A. Bouma, W. vaN DaM. Biochem. Z., 92, 385, 1918. 
North et Park (26) ont fait couler du lait contaminé de bacilles de la tuberculose à travers une spirale de plomb, qui était maintenue avec précision à température constante dans un bain-marie. Ils ont modifié aussi bien la vitesse d'écoulement(et par la la durée de chauffage) que la température, et ils ont recherché, au moyen d'inoculations prati quées à des cobayes, quels étaient les échantillons qui contenaient et quels étaient ceux qui ne contenaient pas de concentration mortelle de bacilles. C'est DAHLBERG (28) qui, le premier, a mis le résultat de ces belles expériences en graphique

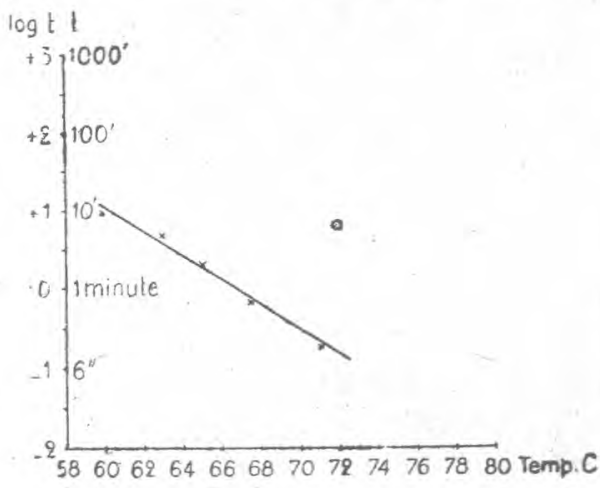

Figure 4a. - Bacilles de la tuberculose juste tués (NoRTH et Park). logarithmique. Il montra qu'une ligne parfaitement droite réunit les points qui ont pour coordonnées les combinaisons précises de températures et de log. temps, qui sont suffisantes pour rendre inof-

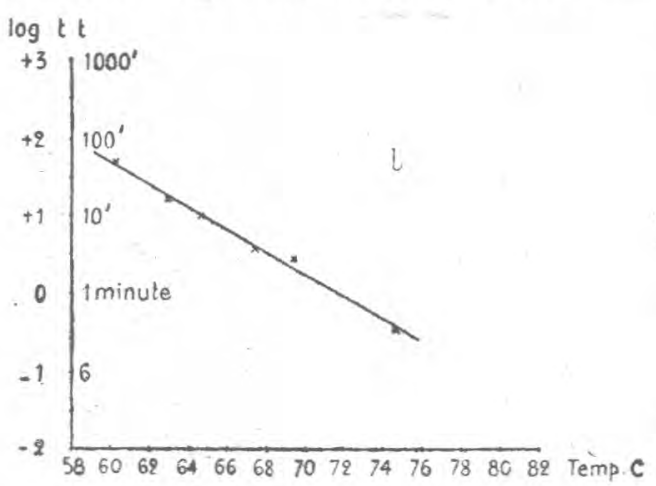

Figure $4 b$. - Phosphatase réduite à $4 \%$ de la valeur initiale (KAY). fensifs les bacilles de la tuberculose du lait dans les expériences de PARK sur des cobayes.

Dalberg $(27,28)$ avait étudié le crémage. Le lait était rapidement chauffé dans un pasteurisateur expérimental, puis maintenuàtempérature constante dans un thermostat. On recherchait quelles étaient les combinaisons de température et de temps exigées afin de conserver exactement le même effet de crémage que celui du lait cru. L'auteur n'a pas dit clairement quelles sont les moindres

$26)$ Ch. E. North, W. H. Park. Amer. Journ. Hyg., 7, 147, 1927.

27 ) J. C. Marquardt, A. C. Dahlberg. N. Y. State Agr. Exp. Stat. Techn. Bull., 180 , juillet 1931 .

(28) A. C. Dahlberg. N.Y. State Agr. Exp. Stat. Tech. Bull., 203, septembre 1932. 
différences entre le lait cru et le lait pasteurisé qu'il a encore reconnues; les déterminations de crémage n'étaient pas très précises.

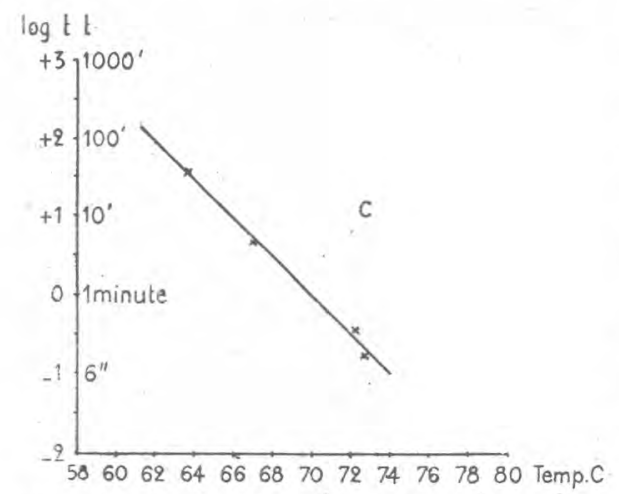

Figure 4c. - Même crémage que celui du lait eru (DAHLBerg).

On n'a pas non plus tenu compte du fait que la vitesse de crémage passe par un maximum lorsque les températures de chauffage augmentent. Le résultat, cependant, est que les points de Dahlberg montraient également une relation linéaire entre $T$ et $\log$. $t$. (fig. $4 \mathrm{c})$.

KAY à son tour renvoya à DAHLBERG et rechercha lui-même quelle était la durée de chauffage nécessaire pour réduire la teneur en phosphatase du lait à $4 \%$ de la valeur initiale (fig. $4 \mathrm{~b}$ ).

On a aussi effectué des recherches sur la thermo- $\log \mathrm{t} l$ labilité des albumines. Zilva (24) avait déjà remarqué que le coefficient de température de $E$ lors du chauffage de la peroxydase correspondait à celui de l'inactivation, par chauffage, de la tétanolysine et de la vibriolysine.

Rowland (29) a recherché comment le pourcentage d'albumine du

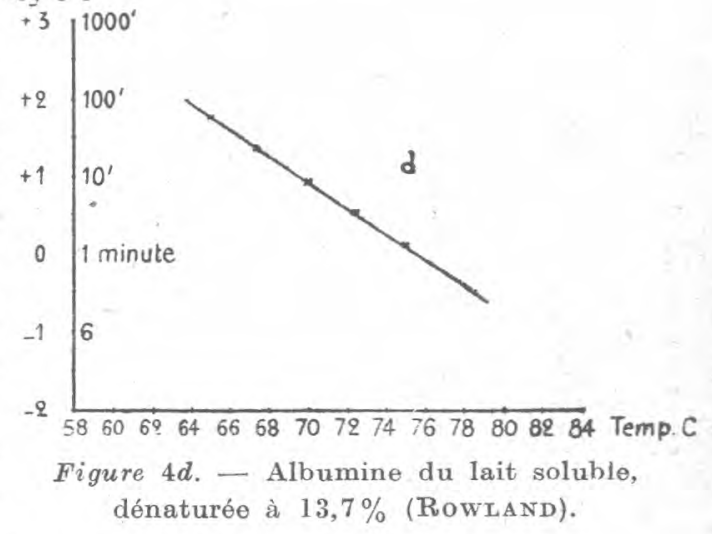
lait diminuait par chauffage. Il publia, lui aussi, un graphique avec $T$ et log. $t$ comme axes, où les points de même concentration se trouvaient sur des droites (fig. 4d). Les lignes de diverses concentrations étaient parallèles. En outre, Rowland montre (ce que $\mathrm{K}_{\mathrm{AY}}$ et DAHLBerg n'avaient pas fait) que la réaction était du premier ordre : précise pour des albumines ịsolées en solution, approxi- 
mative pour l'albumine de lait soluble. KOPPEJAN démontre que la réaction est du premier ordre pour la phosphatase (29a).

Si nous résumons maintenant ce que l'on a écrit sur la base de

\section{$\log t$}

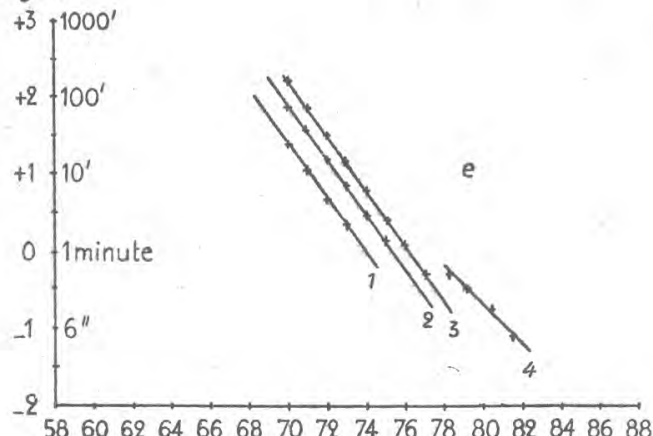

$5860626466 \quad 68 \quad 7072 \quad 747678 \quad 8082848688$ Temp C

Figure 4e. - Pero. xydase. 1. 2. Zilva (50, resp. $90 \%) 3$. (BOUMA et V. DAM) $(99 \%)$ 4. FINCK (annihilee de $90 \%$ ).

Relation entre le logarithme de la durée du chauffage t et la tempéra. ture $T$ lors de diminution de concentration en \% constante pour différentes enzymes et bactéries. (Les ordonnées donnent les valeurs de $t$ avee celles de log t.)

données empiriques, au sujet de la relation entre la température $T$ (en degrés C.), le temps de chauffage $t$ (en minutes) et la concentration (exprimée dans la concentration initiale), nous constatons que :

$10 \log \frac{c_{t=0}}{c_{t}}$ varie proportionnellement à la durée de chauffage $t$ à température constante $T$ pour :

a) La peroxydase ;

b) Les solutions d'albumine isolée ;

en d'autres termes : $\left(d \log \frac{c_{t=0}}{c_{t}} / d t\right)_{\mathrm{r}}=$ constante $\ldots \mathrm{Ia}$, ou bien $\quad \ln \frac{\mathrm{c}_{t=0}}{\mathrm{c}_{t}}=k_{\mathrm{T}} t \ldots \ldots \ldots \ldots$ Ib.

$2^{\circ} T$ diminue proportionnellement à l'augmentation de $\log t$ pour le lait où :

a) La teneur en bacilles de la tuberculose n'est juste plus suffisante pour rendre malades des cobayes;

b) Le crémage est encore juste pareil à celui du lait cru ;

c) La teneur en phosphatase est tombée à $4 \%$ de sa valeur initiale ;

(29a) C. A. Koppejals (Hand. Gen. Melkk., 17, 1936) a trouvé un doublement de kT par degré d'élévation de T entro 58 et $62^{\circ} \mathrm{C}$. A la page 21 , le compositeur a confondu $t$ avec $T$. 
d) La teneur en albumine de lait soluble est tombée à quelques fractions, de sa valeur initiale, en d'autres termes: $-(d \log t / d T)_{\mathrm{c}}=$ const ante, soit $\alpha \ldots \mathrm{IIa}$,

ou bien $k_{\mathrm{c}}=\log t+\alpha T \ldots \ldots \ldots \ldots \ldots \ldots \ldots \ldots$ IIb,

ou $K_{\mathrm{c}}$ peut être une fonction de $c$, mais non de $t$ ou de $T$.

Pour les formules I et II, l'explication est toute prête :

Nous admettons que, lors du chauffage du lait, non seulement la peroxydase et l'albumine dissoute, mais aussi la phosphatase, l'agglutinine et les bacilles de la tuberculose subissent des changements avec une vitesse qui est déterminée par la formule des réactions du premier ordre, e'est-à-dire :

$$
-\frac{1}{c} \frac{d c}{d t}=k_{\mathrm{T}} \ldots \ldots \ldots \ldots \ldots \ldots \ldots \ldots \ldots \text { III, }
$$

où $c$ représente la concentration, $t$ le temps et $k T$ une constante pour la température $T$. Comme on sait, on obtient par intégration :

$$
\ln \frac{c_{t=0}}{c_{t}}=k_{\mathrm{r}} t, \mathrm{c}^{\prime} \text { est la formule Ib. }
$$

Nous admettons encore, que la constante de réaction se comporte comme décrit par la formule d'Arrhenius :

$$
\frac{\mathrm{d} \ln k_{\mathrm{r}}}{d T}=\frac{A}{T^{2}} \ldots \ldots \ldots \ldots \ldots \ldots \ldots \ldots \ldots \ldots \ldots \text { IV }
$$

où $T$ est la température, a bsolue et $A$ une constante, la formule intégrée est :

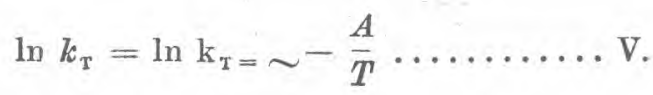

Pour nous tenir aux représentations graphiques de $\mathbf{K A Y}_{\mathbf{A}}$ et DAHLBERG, appliquons une approximation permise, soit :

$$
-\frac{A}{T}=\delta T-\gamma \ldots \ldots \ldots \ldots \ldots \text { VI. }
$$

où $T$ est la température absolue (entre $333^{\circ}$ et $353^{\circ} k$ ) et $\delta$ et $\gamma$ deux nouvelles constantes. En passant des logarithmes naturels à ceux de Briggs, et des températures absolues aux degrés Celsius (que nous désignerons plus loin aussi avec $T$ ), on obtient (en désignant les nouvelles constantes avec $\alpha$ et $\beta$ ) :

$$
\log \log \frac{c_{t=0}}{c}=\log t+\alpha T-\beta \ldots \text { VII. }
$$


d'où suivent la formule II a et en même temps la grandeur $k_{\mathrm{c}}$ dans IIb.

De la formule VII suivent non seulement les formules Ib et IIb, qui donnent la relation entre $\frac{c_{t=0}}{c_{t}}$ et $t$, resp. $T$ et $t$, mais encore une relation entre :

$\frac{c_{t=0}}{c_{t}}$ et $T$, c'est-à-dire : $\left\{d\left(\left(\log \log \frac{c_{t=0}}{c_{t}}\right) / d T\right\}_{\mathrm{t}}=\alpha \ldots\right.$. VIII.

Nous n'avons pas trouvé la relation VIII dans la littérature ni des données suffisamment précises pour vérifier la relation. Jusque maintenant, les moyens d'une vérification directe nous ont manqué. Mais nous avons mesuré la teneur en phosphatase et les vitesses de crémage dans des échantillons de lait, qui étaient pasteurisés (à une vitesse d'écoulement constante), à des températures différentes, dans un même appareil de pasteurisation. Nous avons calculé alors comme suit la relation attendue ici entre la concentration (de la phosphatase, resp. agglutinine) et la température.

Supposons que la température finale, dans un appareil d'écoulement, soit $T_{\mathrm{e}}$, et que l'appareil soit parcouru en $t_{\mathrm{e}}$ minutes. Soit la température à chaque point du tube :

$$
T_{\mathrm{t}}=T_{\mathrm{e}}-\mathrm{f}(\mathrm{t}) \quad \ldots \ldots \ldots \ldots \ldots \ldots \ldots \text { IX. }
$$

On a alors, selon III, l'égalité : $-\frac{1}{c} \frac{d c}{d t}=k_{\mathrm{r}}$.

De V, VI et IX, il suit que : $\alpha \mathrm{T}_{\epsilon}-\mathbf{f}(t)-\varepsilon=\log \mathrm{k}_{\mathrm{T}} \ldots \mathrm{XI}$.

où $\alpha$ et $\varepsilon$ représentent de nouveau des constantes. Après élimination de $\mathbf{k}_{\mathrm{T}}$ et intégration, il vient :

$$
\begin{gathered}
\int_{c_{\mathrm{t}=0}}^{c_{\mathrm{te}}}-d \log c=\int_{0}^{t e} 10^{\alpha T_{\mathrm{e}}-\mathrm{f}(t)-\varepsilon} d t \quad \mathrm{XII} . \\
\log \frac{c_{\mathrm{t}=0}}{c_{\mathrm{t}}}=10^{\alpha T_{\mathrm{e}}-\varepsilon} \int_{0}^{t e} 10^{-\mathrm{f}(\mathrm{t})} d t \quad \text { XIII. } \\
\log \log \frac{c_{\mathrm{t}=0}}{c_{\mathrm{t}}}=\alpha T_{\mathrm{e}}-\varepsilon-\mathrm{F}\left(t_{\mathrm{e}}\right) \ldots \ldots . \mathrm{XIV} .
\end{gathered}
$$

Si dans la pasteurisation avec valeurs terminales différentes $\mathbf{T}_{\mathrm{c}}$ (donc, quand les "températures de pasteurisation" sont différentes) le même $f(t)$ (donc, le même accroissement de température dans le pasteurisateur) subsiste, on doit avoir l'égalité : 


$$
\left\{d\left(\log \log \frac{c_{\mathrm{t}=0}}{c_{\mathrm{t}}}\right) / d T\right\}_{t_{\mathrm{e}}}=\alpha \ldots \ldots \times \mathrm{XV} .
$$

laquelle formule est tout à fait analogue à la formule VIII. Pour une même enzyme ou bactérie, $\alpha$ doit être numériquement égal pour le chauffage constant (VIII) et pour le chauffage par écoulement (XV).

Il a paru en effet (fig. 5) que, lorsque les résultats de nos déterminations de phosphatase furent représentés graphiquement sur axes $\log \log \frac{c_{\mathrm{t}=0}}{c_{\mathrm{t}}} \sim \mathrm{T}$, les points se trouvaient tout près d'une ligne droite, avec un éparpillement qui était en concordance avec les erreurs de la manipulation et de la température.

On a pu observer aussi, bien qu'avec approximation, une même relation entre la température et la concentration de l'agglutinine $\mathrm{du}$ crémage. Nous avons recherché dans notre figure Ib les pourcentages de lait cru $p$, pour lesquels l'épaisseur de crème après 8 heures était aussi grande que pour les échantillons de lait pasteurisés à température terminale de pasteurisation $T_{\mathrm{e}}$ connue. Il a paru de nouveau que $\log$. $\log 1 / \mathrm{p}$, comparé à $\mathrm{T}_{\mathrm{e}}$, donnait des points qui se trouvaient très près d'une droite (fig. 1d).

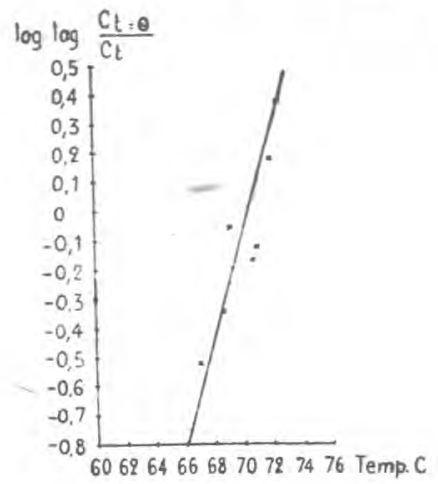

Figure 5. - Résultats de déterminations de phosphatase dans du lait, préalablement chauffé à $70^{\circ} \mathrm{C}$. dans un appareil à courant continu et chauffé ensuite en 1 seconde 4 à la température finale To C. $; \frac{c_{\mathrm{t}=0}}{c_{\mathrm{t}}}=$ concentration de phosphatase dans le lait eru/concentration de phosphatase dans le Jait chauffé.

Les déductions montrent donc que te comportement, lors du chauffage d'albumine dissoute, d'enzymes (peroxydase, phosphatase), d'agglutinine et de bacilles de la tuberculose, tel que cela est décrit dans la bibliographie, s'accorde avec : $1^{\circ}$ la rigueur approximative de la formule de vitesse de réaction du premier ordre, et $2^{\circ}$ la loi d'Arrhenius. Elles montrent aussi que en partant de $1^{\circ}$ et $2^{\circ}$ une relation se laisse déduire entre le changement relatif de la concentration et la température pour chaque durée de chauffage, aussi bien pour un chauffage constant que pour un chauffage réalisé dans un appareil à courant continu. Dans le dernier cas, la formule est en concordance avec le résultat des 
épreuves effectuées par nous (résultats de la phosphatase, et, par approximation, du crémage).

La vraisemblance des suppositions, qui sont à la base de l'établissement de la formule VII, est encore confirmée par des données expérimentales, dont le rapport avec elles était moins simple : KAY, par exemple, a recherché le temps $t$ de séjour à l'étùve à $37^{\circ} \mathrm{C}$. d'échantillons de lait (pasteurisés pendant 30 minutes à différentes températures $T$ ) en solution de substrat tamponné, nécessaire pour que le phénol séparé livràt toujours 2 unités 3 de Lovibond en bleu après réaction avec le réactif de Folin. La publication de KAY (13) montrait pour la fonction $t=f(T)$ une courbe de forme hyperbolique. On peut admettre (et nous en avons déjà fait usage dans la note (16)), que la formation de phénol par unité de temps est proportionnelle à tout moment à la teneur en phosphatase. On doit eonsidérer que la teneur en phosphatase diminue d'abord (selon la formule III) pendant la pasteurisation de 30 minutes à $T^{\circ}$ et ensuite dans l'étuve à $37^{\circ}$ pendant $t$ minutes. On a l'égalité :

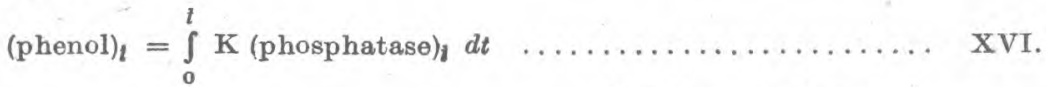

$$
\begin{aligned}
& \left.(\text { phosphatase })_{t}=\text { (phosphatase }\right)_{\mathrm{cru}} \cdot 10^{-\mathrm{k}_{\mathrm{T}} \cdot 30} \cdot 10^{-\mathrm{k}_{37} \cdot \mathrm{t}} \cdot \ldots \mathrm{XVII} .
\end{aligned}
$$

d'où suit par substitution de XVII dans XVI :

$$
\begin{aligned}
& (\text { phénol) })_{t}=K \cdot 10^{-\mathbf{k}_{\mathrm{T}} \cdot 30} \text { (phosphatase) }_{\text {cru }} \int_{0}^{t} 10^{-\mathrm{ks} 9 \cdot \mathrm{t}} \ldots \mathrm{XVIII} . \\
& (\text { phénol })_{t}=\frac{K}{k_{37}}(\text { phosphatase }) \mathrm{cru} \cdot 10^{-\mathbf{k}_{\mathrm{T}} 30}\left(1-10^{-\mathrm{kar}^{\mathrm{t}}}\right) \ldots \mathrm{XIX} \text {. }
\end{aligned}
$$

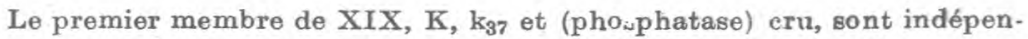
dants de $t$ et de $T$. Après avoir ramené à une constante 105 , il vient :

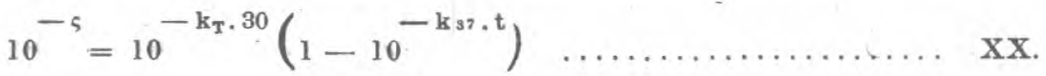

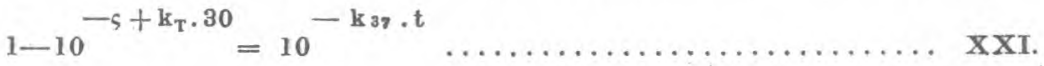

$$
\begin{aligned}
& \frac{1}{2.3} \ln \left(1-10^{-\varsigma+k_{\mathrm{T}} \cdot 30}\right)=-k_{\mathrm{g} \gamma} \cdot t \quad \ldots \ldots \ldots \ldots \ldots \ldots \ldots \ldots \ldots \times \ldots \ldots I .
\end{aligned}
$$

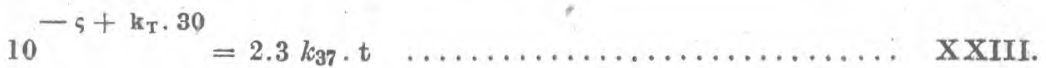

Remplaçons $k_{\mathrm{r}}$ par $10{ }^{\alpha \mathrm{T}}-\beta$, résumons les paramètres indépendants de $t$ et $\boldsymbol{T}$ dans une nouvelle constante $x$ et calculons de nouveau les logarithmes, il vient :

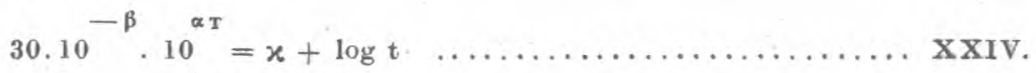

Il nous a paru, en effet, que les observations de $\mathrm{KAY}$ se trouvaient être sur 
une ligne droite, quand log t était porté en ordonnées et $10^{\alpha_{\mathrm{T}}}$ en abscisses, tandis que suivait de l'inclinaison de la ligne (fig. 6) une valeur de $\beta=10.7$, en concordance avec les valeurs obtenues au moyen de la formule VII (tableau D). Des graphiques avec $t$ et $10^{\alpha \mathrm{T}}$, ou $T$ et $\log t$ comme axe donnèrent par contre des lignes courbes.

Au moyen des figures $4 \mathrm{a}-4 \mathrm{e}, 1 \mathrm{~d}, 5$ et 6 , purent être calculées les valeurs pour les paramètres $\alpha$ et $\beta$, qui se présentent dans la formule VII $: \log \log \frac{c_{t=0}}{c_{t}}=\log t+\alpha \mathrm{T}-\beta$. Elles sont données dans le tableau D.

Les chiffres montrent une concordance entre les valeurs $\alpha$, trouvées avec la formule IIa au moyen des données extraites de

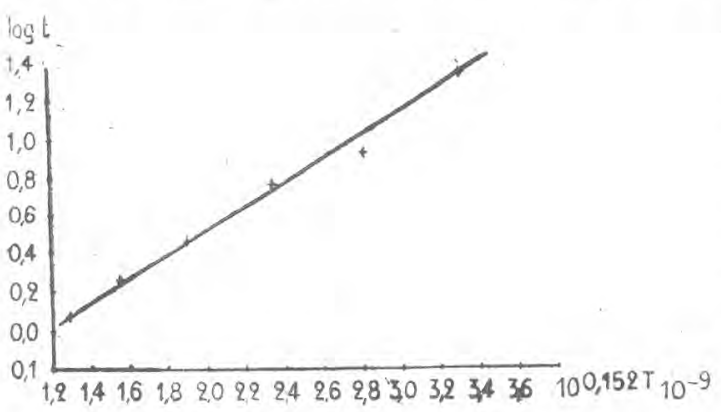

Figure 6.

Relation linéaire entre $\log t$ et $10^{\alpha} \mathrm{T}$, où $T$ est la température de pasteurisation d'échantillons de lait, qui furent traités par KAY è $37^{\circ}$, avec du di-phénylphosphatede sodium, pendant un temps $t$ tel que les mélanges avec le réactif de Folin-Ciocalteu donnèrent la même coloration bleue : $\alpha=0,152$ : durée de pasteurisation toujours ía même.

travaux de différents auteurs (peroxydase), entre les valeurs $\alpha$, calculées avec les formules XV et IIa (agglutinine, phosphatase), entre des valeurs $\beta$ (de la phosphatase), calculées de trois façons différentes.

Enfin, le tableau D nous apprend que, lors du chauffage du lait, la disparition de la peroxydase, de l'albumine soluble, du faeteur activant le crémage et des bactéries, ne correspondent pas seulement qualitativement dans leur comportement (réaction du premier ordre, loi d'Arrhenius), mais aussi quantitativement : de toutes les substances nommées, sauf la peroxydase, la valeur a est pratiquement égale, et, en ce qui concerne la peroydase, elle est du même ordre de grandeur. ( $\mathrm{Vu}$ que toutes les substances ici réunies ont l'albumine comme composant, une explication possible nous semble être que, dans tous ces processus, une insolubilisation de l'albumine est la cause de la thermolabilité.)

Il vaut peut-être la peine de rechercher la validité de la formule VII pour d'autres enzymes, albumines et bactéries qui n'ont pas été envisagées ici $(29 b)$.

(29b) Voir A. K. van Bever, Enzymologia, 11, 7, 1943 (en anglais). 
TABLEAU D

GRANDEURS DES PARAMETRES $\alpha$ ET $\beta$ DE LA FORMULE VII

\begin{tabular}{|c|c|c|c|c|c|c|}
\hline & \multicolumn{4}{|c|}{ Paramètre } & \multirow{2}{*}{\multicolumn{2}{|c|}{ Auteurs des données expérimentales }} \\
\hline & \multicolumn{2}{|r|}{$\alpha$} & \multicolumn{2}{|r|}{$\beta$} & & \\
\hline & Valeur & Calculée de & Valeur & Calculée de & Nom & Note $n^{\circ}$ \\
\hline Peroxydase & $\begin{array}{l}0,35 \\
0,35\end{array}$ & $\begin{array}{l}\text { IIa, fig. } 4 \mathrm{e} \\
\text { IIa, fig. } 4 \mathrm{e}\end{array}$ & $\begin{array}{l}26,5 \\
26,0\end{array}$ & $\begin{array}{l}\text { VII, } \alpha, \text { fig. } 4 \mathrm{e} \\
\text { VII, } \alpha \text { fig. } 4 \mathrm{e}\end{array}$ & $\begin{array}{l}\text { ZILVA } \\
\text { BOUMA, VAN DAM }\end{array}$ & $\begin{array}{l}24 \\
25\end{array}$ \\
\hline $\begin{array}{l}\text { Albumine soluble } \\
\text { du lait }\end{array}$ & 0,17 & IIa, fig. $4 \mathrm{~d}$ & 13,8 & VII, $\alpha$, fig. $4 \mathrm{~d}$ & ROWLAND & 29 \\
\hline Agglutinine & $\begin{array}{r}0,17 \\
0,19\end{array}$ & $\begin{array}{l}\text { IIa, fig. } 4 \mathrm{c} \\
\mathrm{XV} \text {, fig. 1d }\end{array}$ & $12, \dot{4}$ & VII, $\alpha$, fig. id & $\begin{array}{l}\text { DalHBerg } \\
\text { (expérience personnelle de } \\
\text { mélange et de chauffage) }\end{array}$ & $27-28$ \\
\hline Phosphatase & $\begin{array}{l}0,15 \\
0,17\end{array}$ & $\begin{array}{l}\text { IIa, fig. } 4 \mathrm{~b} \\
\mathrm{XV} \text {, fig. } 5\end{array}$ & $\begin{array}{l}10,6 \\
10,4 \\
10,7\end{array}$ & $\begin{array}{l}\text { VII, } \alpha, \text { fig. } 4 b \\
\text { VII, } c_{t}=o, t \\
\text { T, } \alpha, c_{t} \\
\text { XXIV, fig. } 6\end{array}$ & $\begin{array}{l}\text { KAY } \\
\text { Exp. pers. de chauffage } \\
\text { KAY, tabI. II } \\
\text { KAY }\end{array}$ & $\begin{array}{l}13 \\
13\end{array}$ \\
\hline $\begin{array}{l}\text { Bacilles de la } \\
\text { tuberculose }\end{array}$ & 0,17 & Ira, fig. $4 a$ & $\begin{array}{c}10,2 \\
\grave{a} \\
10,5\end{array}$ & $\begin{array}{c}\text { VII, } \alpha, \text { fig. } 4 \mathrm{a} \\
\text { pour } \\
\frac{\mathrm{c}_{\mathrm{t}=0}}{\mathrm{c}_{\mathrm{t}}}=10^{-3} \mathrm{a} \\
10^{-6}\end{array}$ & North, PARK & $26-28$ \\
\hline
\end{tabular}

\section{B. Signification pratique des relations déduites, pour l'appréciation du lait}

Nous résumons maintenant ce qui, dans les phénomènes décrits ci-dessus, est important pour la pratique :

L'influence du chauffage du lait sur la concentration des enzymes, albumines et bactéries, est déterminée par la température de "chauffage " et la durée de celui-ci. A condition que cette dernière soit mesurée en logarithmes, les deux grandeurs jouent un rôle d'égale valeur. Nous considérons maintenant un échantillon de lait cru, contaminé de germes pathogènes, et nous le divisons en portions 1, 2, 3, etc... Nous chauffons ces portions, toutes pendant un même temps $t$, mais la portion 1 à la température constante $\mathbf{T}_{1}$, 
la portion 2 à la température plus élevée $\mathrm{T}_{2}$, la portion 3 à la température encore plus élevée $\mathrm{T}_{3}$, etc...

Si nous déterminons, après ce traitement, la concentration $\mathrm{c}_{\mathbf{t}}$ des bactéries et des enzymes, nous trouvons que, dans les portions ayant subi le chauffage le plus faible, toutes les sortes de particules ont pratiquement conservé leur concentration, dans une portion suivante, par exemple, la concentration en Bacterium Coli est fortement tombée, tandis que les concentrations restantes sont intactes; dans celle qui suit, le Coli est pratiquement annihilé ; le bacille de la tuberculose est un peu affaibli ; ̀̀ des températures plus élevées nous ne trouvons plus ni Coli ni bacilles de la tuberculose ; en continuant, nous trouvons une forte diminution, puis la disparition de la phosphatase, puis de la même manière la disparition du pouvoir crémant et enfin celle de l'enzyme peroxydase (voir les valeurs ascendantes de $T$ pour de petites valeurs de $c_{t}$ à une même durée de chauffage $t$, par exemple $\mathrm{t}=1$ minute, allant de la figure $4 \mathrm{a}$ à $4 \mathrm{~b}$ jusque $4 \mathrm{e}$ ). On lit quantitativement l'ordre de succession de l'annihilation quand la température augmente, dans l'augmentation du paramètre $\beta$ de la formule VII (tableau D).

Si nous examinons de nouveau la série de nos portions chauffées, et si nous faisons attention, dans chaque cas particulier $\mathrm{t}_{\mathrm{t}}$ au changement de la concentration terminale $\mathrm{C}_{t}$ avec la température de chauffage $T$, nous voyons que les "tranches" de température au dedans desquelles la teneur en phosphatase, le pouvoir crémant, la teneur en peroxydase, etc... tombent d'un pourcentage élevé à un pourcentage restreint (par exemple de $70 \%$ à $7 \%$ ) de la valeur originale sont petites. Cela signifie que l'on a affaire ici à une zone de variation rapide. Cette propriété se lit immédiatement dans notre formule VII :

$$
\log \log \frac{c_{\mathrm{t}=0}}{c_{\mathrm{t}}}=\log t+\alpha \mathrm{T}-\beta
$$

$\mathrm{Si}$ nous exprimons $\mathrm{C}_{\mathrm{t}} / \mathrm{C}_{\mathrm{t}}=0$ (c'est-à-dire la concentration terminale divisée par la concentration initiale) en puissances de 0,1 l'exposant de cette puissance (si $\alpha=1 / 6$, comme pour la phosphatase, le crémage et le bacille de la tuberculose) est alors multiplié par 10 pour chaque tranche de 6 degrés d'élévation de température quand $\alpha$ reste constant (tableau $\mathrm{E}$ ). 
TABLEAU E

TABLEAU GHIFFRE EN ILLUSTRATION DE LA FORMULE VII

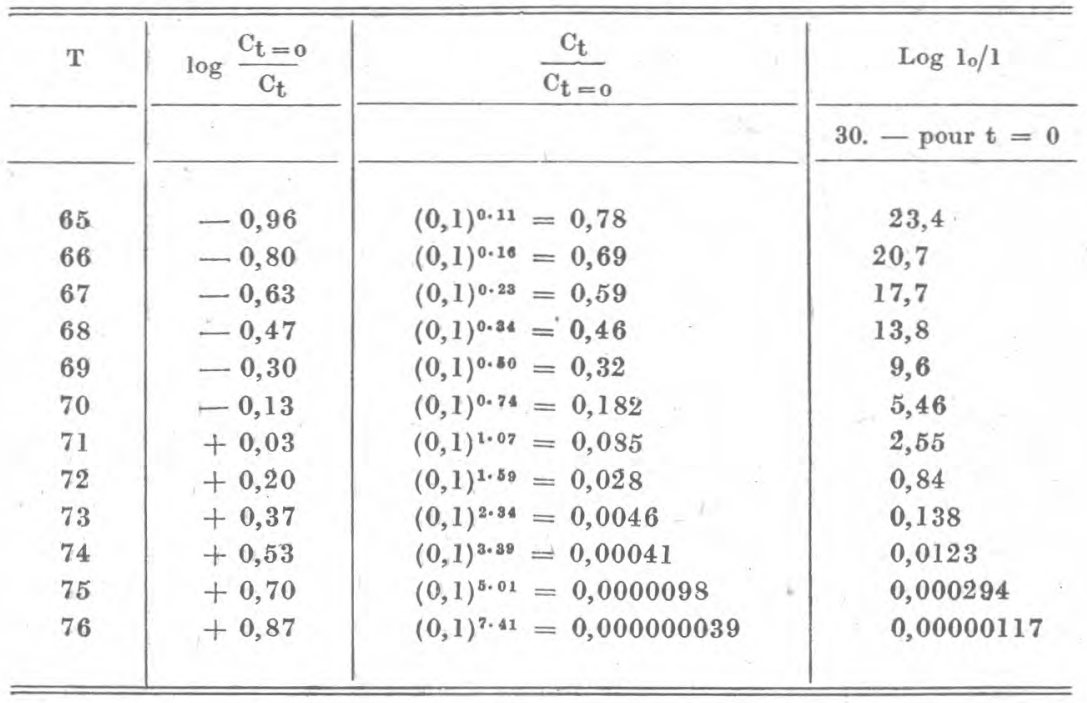

$t=$ durée de pasteurisation constante de $\frac{1,4}{60}$ minute.

$\mathbf{T}=$ température de pasteurisation en degrés $\mathbf{C}$.

$c_{t}=0=$ concentration de la phosphatase avant chauffage.

$c_{t}=$ concentration de la phosphatase après chauffage

$\log \mathrm{I}_{0} / \mathrm{I}=$ valeur calculée de la concentration de phosphatase telle qu'on l'obtient, si elle est déterminée au Stufomètre et si le lait non chauffé donnait 30.-

Les chiffes sont calculés au moyen de la formule $\log \log \frac{c_{t}=0}{c_{t}}$ $=\log \mathrm{t}+1 / 6 \mathrm{~T}-10,17$.

(Les paramètres $\alpha=1 / 6$ et $\beta=10.17$, choisis ici, sont ceux qui correspondent aux épreuves de pasteurisation de la fig. 5.)

Comme exemple, on a choisi la phosphatase. Que l'on compare, par exemple $\frac{c_{t}}{c_{t=0}}=(0,1)^{0.16}$ pour $T=66^{\circ} \mathrm{C}, t \pm=1,4^{\prime \prime}$, avec $\frac{\mathrm{c}_{t}}{\mathrm{c}_{t=0}}=(0,1)^{1 \cdot 6}$ pour $T=72^{\circ} \mathrm{C} ., t=1,4^{\prime \prime}$.

La figure 7 donne la représentation graphique.

Si nous chauffons du lait, dans une nouvelle série d'essais, $g$ fois aussi longtemps, done gt minutes, il se produit dans les portions successives portées à une température toujours plus élevée les mêmes phénomènes que tout à l'heure, avec cette différence que 
chaque variation a lieu à une température de $\frac{1}{\alpha} \log g$ degrés plus bas que lors du premier temps de chauffage. S le temps de chauffage

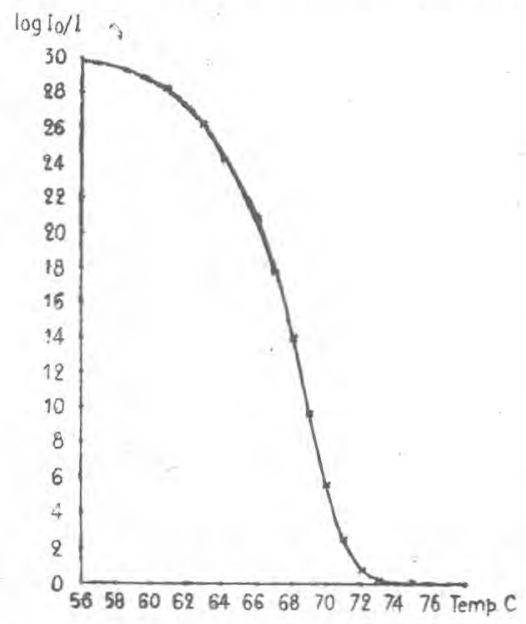

Figure 7. - Lectures au Stufomètre, calculées. des teneure en phosphatase du lait pasteurisé pendant $1,4^{\prime \prime}$ à $\mathrm{T}^{\circ} \mathrm{C}$., d'après les données du tableau E, $4^{\mathrm{e}}$ colonne.

ne change pas dans de orandes limites de temps de chauffage. (VAN EcK (23) a remarqué très justement en 1911 déjà que la possibilité d'emploi de la réaction de Storch dépend de la rigueur de cette règle.)

La pratique apprend que l'ordre de succession décrit plus haut subsiste quand les temps de chauffage sont réduits à quelques secondes, et par là, la validité des conclusions que nous tirons des mesures de la phosphatase, du crémage et de la réaction de Storch.

\section{III. - LE CONTROLE DU LAIT " SOUS PASTEURISÉ " AU MOYEN DE L'ÉPREUVE DE LA PHOSPHATASE}

Pour le contrôle du lait, nous nous servons de ce qui précède de la manière suivante : on conclut du résultat négatif de l'épreuve de phosphatase (sans que la vitesse d'écoulement et la température de pasteurisation soient connues), que le chauffage a été suffisant pour tuer les germes pathogènes ( $y$ compris les bacilles de la tuberculose) et cela aussi quand le crémage et la peroxydase subsistent. Un pouvoir de crémage diminué témoigne d'un chauffage plus fort (en ce cas, la présence d'un peu de phosphatase témoigne de l'addition de lait insuffisamment ou non chauffé). Nous concluons à un 
degré de chauffage encore plus élevé, quand la réaction de Storch donne un résultat négatif; la vitesse de crémage est alors tellement restreinte qu'il devient, en un jour, impossible de la mesurer (ici aussi une réaction positive de phosphatase témoigne de la présence d'un pourcentage de lait beaucoup moins chauffé).

Il suit de la disposition des lignes du log. $t \sim T$ des bacilles de la tuberculose et de la phosphatase (fig: $4 a$ et $4 b$ ), que la détermination de la phosphatase répond aux exigences que nous avons posées à un contrôle chimique de la pasteurisation basse : si la phosphatase est absente du lait, c'est que la température de pasteurisation a été un peu plus élevée qu'il n'est nécessaire (pour une même durée de chauffage) pour anéantir les bacilles de la tuberculose, mais qu'elle a été plus basse qu'il n'est nécessaire pour donner un résultat négatif de la réaction de Storch.

Il est vrai, pour chaque vitesse d'écoulement, que la phosphatase disparaît à une température d'autant plus élevée que, par exemple, les bacilles de la tuberculose, et d'autre part, qu'elle disparaît, pour chaque température de pasteurisation, à un multiple aussi grand de la durée de chauffage.

Exemple: pour un chauffage d'une durée de une minute, il faut, pour une réaction de Storch négative, une température de 6 degrés environ plus élevée que pour une valeur zéro de la détermination de la phosphatase. Pour que la phosphatase, aussi bien que les bacilles de la tuberculose, soient annihilés, un changement de température de $6^{\circ} \mathrm{C}$. répond à une durée de chauffage décuplée.

Il est nécessaire de se rendre compte jusqu'à quel point on peut faire confiance, en général, à la règle selon laquelle les bacilles de la tuberculose sont tués quand la pasteurisation a annihilé la phosphatase.

Nous avons trouvé la règle confirmée, lorsque, lors d'une épreuve de contrôle, le Service d'inspection, ayant composé du lait fortement contaminé de bacilles de la tuberculose et l'ayant "sous-pasteurisé » à des températures différentes, examina les échantillons prélevés au point de vue de leur teneur en bacilles de la tuberculose (sur des cobayes) et en phosphatase : la différence entre la température d'annihilation des bacilles et celle de la phosphatase était suffisamment grande pour que l'on pût estimer "sans danger o du lait exempt de phosphatase provenant de cet appareil. Il faut pourtant ne pas perdre de vue ce qui suit :

$1^{\circ}$ Des courbes telles que les lignes de $\log t \sim T$ et des constantes telles que $\alpha$ et $\beta$ sont propres à éclaircir la relation des phénomènes. L'inclinaison et l'emplacement de ces courbes, ou - ce qui revient au même - la grandeur de $\alpha$ et $\beta$, sont cependant affectés d'incerti- 
tude à la suite de l'imprécision inévitable des concentrations des enzymes et des températures de pasteurisation et surtout des épreuves biologiques (recherches sur les cobayes), d'où elles sont calculées. Il n'est pas certain, non plus, que les bacilles de la tuberculose ne sont jamais plus résistants à la chaleur que lors des épreuves de North et Park. En tout cas, les germes de la tuberculose en fortes concentrations sont plus difficiles à tuer qu'en petites concentrations, et ils sont plus résistants lorsqu'ils sont renfermés dans des particules solides que lorsqu'ils se meuvent librement dans le lait ou dans l'eau ;

$2^{\circ}$ Les lignes de $\log t \sim T$ des bactéries et des enzymes se couperont si les $\alpha$ ne sont pas parfaitement égaux (et cela d'autant plus tôt que les $\alpha$ seront plus différents entre eux et que les $\beta$ le seront moins), si bien que les conclusions (par exemple : «les bacilles de la tuberculose sont tués, parce que le résultat de la phosphatase est négatif ») ne sont plus justifiés pour des durées de chauffage encore plus courtes que celles des épreuves (30). Cela vaut aussi pour des degrés de température qui n'ont pas été étudiées ;

$3^{\circ}$ Ce n'est pas seulement lors de l'épreuve au laboratoire, mais, en outre, lors de la pasteurisation industrielle que les circonstances - température de pasteurisation, vitesse d'écoulement et teneur en bactéries du lait eru - peuvent présenter des fluctuations. Pour ces motifs, la marge de sécurité entre l'annihilation des bacilles de la tuberculose et de la phosphatase (exprimée en degrés ou en multiples de la durée de chauffage) est plus petite que la distance moyenne, que l'on peut calculer après une série d'épreuves, ou que l'on peut lire sur nos graphiques. C'est pourquoi il est absolument nécessaire de prendre les précautions suivantes (pour leur exécution, voir pages 1 et suivantes) :

$1^{0}$ Etablissement bactériologique de la marge "phosphatasebacilles de la tuberculose "lors de l'installation de nouveaux types d'appareils ;

$2^{\circ}$ Maintien, aussi large que possible, de la marge entre la mort des bactéries et l'annihilation des enzymes, en:

a) Maintenant la température et la vitesse d'écoulement reconnues sûres lors $d u 1^{\circ}$;

b) Evitant des taux bactériens trop élevés dans le lait cru;

c) Limitant à l'extrême les fluctuations de température et de vitesse d'écoulement, nettoyant fréquemment et maintenant en

(30) Sur ce temps de chauffage réduit, et d'autres données sur un appareil de pasteurisation moderne, consulter : "Translation of the 10th Report from the Danish Government Experimental Dairy (Ringkjohing, 1938). 
bon état l'appareil et les instruments (contrôle officiel de tout ceci par l'inspection technique).

$3^{\circ}$ Opérant un contrôle bactériologique constant du lait par le fabricant lui-mếme et par l'autorité (total du nombre de bactéries, colibacilles, de temps en temps recherche biologique de l'absence du bacille de la tuberculose) ;

$4^{\circ}$ Prenant les dispositions nécessaires pour obtenir un produit exempt de phosphatase. C'est aux autorités d'exiger ceci et de contrôler l'observation de cette prescription avec la méthode la plus précise.

Si l'on travaille d'après ce schéma, la mesure de la concentration de l'enzyme comme indication des déviations dans les conditions de pasteurisation établies sera une partie utile et indispensable du contrôle du lait sous-pasteurisé, et la méthode à suivre sera la détermination de phosphatase de Kay.

Nous considérons comme inadmissible le critérium choisi par $\mathrm{K}_{A Y}$ pour la bonne pasteurisation du lait. $K_{A Y}$, en effet, est satisfait si le résultat total de la phosphatase (done de la détermination $-\mathrm{S}$, voir I-3 ${ }^{\circ}$, page 13 ) est inférieur à 2,3 unités de Lovibond-bleu. Au lieu de soustraire de l'as l'effet de substances phénoliques dans le lait, au moyen d'une détermination $W$ dans le même échantillon, $\mathrm{K}_{\mathrm{AY}}$ soustrait une correction fixe, à savoir une quantité $(2,3$ unités de Lovibond-bleu), qui est déjà trop grande pour une valeur moyenne de $a_{\mathrm{w}}$ et qui l'est d'autant plus pour des valeurs de $a_{\mathrm{w}}$ restreintes. C'est par là que la prescription anglaise permet expressément une quantité déterminée de lait cru (environ $0,3 \%$ ).

L'exigence, qu'il faut formuler, c'est qu'on ne puisse (mais pas du tout) montrer la présence de phosphatase dans du lait, pasteurisé, en bouteilles ( 31 ).

Cette exigence interdit complètement la présence de lait cru, ce qui est d'une nécessité urgente pour la sécurité publique.

Parfois, les exploitants d'entreprises de pasteurisation objectent, au désir que l'épreuve de phosphatase ait un résultat négatif, qu'un tel chauffage ferait diminuer la vitesse de crémage, ce qui provoquerait des plaintes de la part des clients.

Cette objection n'est pas du tout fondée. Le tableau F - à augmenter d'une multiplicité de chiffres - montre qu'une teneur

(31) Déjà, dans les Handelingen Gen. Melkk., 1939 (Exposé de Ir. J. Straub, en collaboration avec A. K. VAN BEver), on a montré que le fait que P. L. SutherLAND (Journ. Roy. Sanit. Inst., 58, 693, 1937.1938) a trouvé des bacilles de la tuberculose dans beaucoup de laits prétendus exempts de phosphatase, doit être imputé, non seulement à des contaminations ultérieures, mais en général à l'exigence insuffisante de Kay. 
pratiquement nulle en phosphatase dans le lait d'installations amsterdamoises peut très bien aller de pair avec un crémage égal ou plus élevé que celui du lait cru.

L'installation de pasteurisation, qui veut conserver le crémage, se pose par là, à soi-même, l'exigence d'éviter des fluctuations de température plus grandes que $\pm 0^{\circ} 5 \mathrm{C}$., vu que la distance entre les courbes de $\log t \sim T$ pour la phosphatase et la conservation du crémage n'est pas grande. Il peut être facilement satisfait à cette exigence si l'homme à l'appareil est suffisamment exercé et s'il

\section{TABLEAU F}

GREMAGE RAP IDE LORS DE L'ABSENGE DE PHOSPHATASE DANS LE LAIT " SOUS-PASTEURISÉ "

\begin{tabular}{|c|c|c|c|c|c|}
\hline \multirow[b]{2}{*}{ Mois } & \multirow[b]{2}{*}{$\begin{array}{l}\text { Traitement } \\
\text { préalable }\end{array}$} & \multirow[b]{2}{*}{$\begin{array}{c}\text { Eehantillon } \\
\text { de lait }\end{array}$} & \multirow[b]{2}{*}{$\begin{array}{c}\text { Phosphatase } \\
\log I_{0} / 1 \text { pour } \\
\mathrm{d}=0,5 \mathrm{~cm} \\
\lambda=610 \mu\end{array}$} & \multicolumn{2}{|c|}{$\begin{array}{c}\text { Crémage en colonne } \\
\text { de } 20 \mathrm{~cm} .\end{array}$} \\
\hline & & & & $\begin{array}{l}\text { Epaisseur } \\
\text { de crème } \\
\text { après } 1 \mathrm{~h} ., \\
\text { en centi- } \\
\text { mètres }\end{array}$ & $\begin{array}{c}t_{1} \% \\
\text { (nombre } \\
\text { d'heures } \\
\text { avant } \\
\text { d'attein. } \\
\text { dre } \\
0,2 \mathrm{~cm} .\end{array}$ \\
\hline Novembrel 939 & cru & $\mathrm{S}_{1}$ & +32. & 0,60 & 0,3 \\
\hline . & pasteurisé & $\begin{array}{l}\text { B } 310 \\
\text { B } 307 \\
\text { B } 311 \\
20499 \\
20575 \\
21216\end{array}$ & $\begin{array}{r}-0,02 \\
-0,01 \\
0,00 \\
+0,02 \\
+0,02 \\
+0,03\end{array}$ & $\begin{array}{l}1,05 \\
1,00 \\
1,20 \\
0,55 \\
1,15 \\
1,00\end{array}$ & $\begin{array}{l}0,2 \\
0,2 \\
0,1 \\
0,4 \\
0,2 \\
0,2\end{array}$ \\
\hline Décembre 1939 & eru & R $5 \mathbf{a}$ & $+38 .-$ & 0,55 & 0,4 \\
\hline & pasteurisé & $\begin{array}{l}21878 \\
\text { B } 349 \\
22582\end{array}$ & $\begin{array}{r}+0,01 \\
0,00 \\
0,00\end{array}$ & $\begin{array}{l}1,05 \\
1,30 \\
0,45\end{array}$ & $\begin{array}{l}0,2 \\
0,2 \\
0,4\end{array}$ \\
\hline Février-mars & cru & 4809 & $+34 .-$ & 0,60 & 0,3 \\
\hline & pasteurisé & $\begin{array}{l}2067 \\
2261 \\
2563 \\
3065\end{array}$ & $\begin{array}{r}+0,01 \\
+0,01 \\
0,00 \\
-0,04\end{array}$ & $\begin{array}{l}0,70 \\
0,55 \\
0,80 \\
0,85\end{array}$ & $\begin{array}{r}0,3 \\
0,4 \\
0,3 \\
0,2\end{array}$ \\
\hline
\end{tabular}


n'est pas empêché d'exercer une surveillance constante du chauffage par d'autres activités ou par une disposition peu claire des instruments. Il va de soi que la vitesse d'écoulement ne doit pas varier non plus.

Afin de donner une vue de l'exécution pratique de la surveillance de la pasteurisation, disons encore qu'à Amsterdam le lait, en bouteilles, "Voltana » et " Stassano » est vendu par cinq installations. Celles-ci sont inspectées chaque semaine; leurs thermomètres sont contrôlés et les bandes de thermomètres auto-enregistreurs sont vérifiées. Chaque jour, on prélève en ville des échantillons du lait de ces entreprises, et on les soumet à un examen approfondi. Les tableaux $F$ et $G$ donnent une image des résultats ainsi obtenus.

TABLEAU G

RÉSULTATS D'UNE RECHERCHE JOURNALIËRE

\begin{tabular}{|c|c|c|c|c|c|}
\hline $\begin{array}{l}\text { No de } \\
\text { l'ana. } \\
\text { lyse }\end{array}$ & Fournisseur & $\begin{array}{c}\text { Phosphatase } \\
\log 1_{0} / 1 \\
d=0,5 \\
\lambda=610\end{array}$ & $\begin{array}{l}\text { Storch } \\
\text { quali- } \\
\text { tatif }\end{array}$ & $\begin{array}{c}\text { Storch } \\
\text { quantitatif } \\
\log 1_{0} / 1 \\
d=3 \\
\lambda=\mathbf{5 3 0}\end{array}$ & $\begin{array}{c}\text { Crémage } \\
t_{1 \%} \%\end{array}$ \\
\hline 3643 & sous-pasteurisé I & $+0,02$ & + & $+6,65$ & 1,2 \\
\hline 3646 & $" \quad$ II & $-0,01$ & + & $+5,39$ & 1,4 \\
\hline B 73 & III & $+0,01$ & + & $+5,64$ & 1,2 \\
\hline B 96 & III & $+0,01$ & + & $+5,13$ & $>7 .-$ \\
\hline 3722 & I & 0,00 & + & $+7,57$ & 0,60 \\
\hline 3723 & III & 0,00 & + & $+5,08$ & $>7 .-$ \\
\hline 3727 & IV & 0,00 & + & $+6,32$ & 1,8 \\
\hline $\begin{array}{r}9 \\
10\end{array}$ & $\begin{array}{c}\text { lait écrémé, appa. } \\
\text { reil ancien }\end{array}$ & $\begin{array}{l}+0,11 \\
-0,01\end{array}$ & $\begin{array}{l} \pm \\
\pm\end{array}$ & $\begin{array}{r}+0,63 \\
+0,61\end{array}$ & \\
\hline 3779 & sous-pasteurisé III & 0,00 & + & $+7,66$ & $>7 .-$ \\
\hline 3781 & $n \quad I$ & $-0,01$ & + & $+7,45$ & 1,1 \\
\hline 2884 & surpasteurisé I & & - & $+0,02$ & \\
\hline 2886 & II & & - & $+0,13$ & \\
\hline .2897 & III & & 一 & $+0,04$ & \\
\hline 2899 & III & & 一 & $+0,07$ & \\
\hline 2900 & $n \quad$ IV & & & $-0,03$ & \\
\hline
\end{tabular}

Les exemples suivants montrent comment ces mesures ont amené la découverte d'erreurs :

Une installation livre depuis quelques mois du lait exempt de 
phosphatase $(-0,03$ à $+0,03)$ avec un crémage pareil à celui du lait cru $\left(t_{1 \%}=0,3 \grave{a} 1,0\right)$. Les diagrammes thermométriques montrent des fluctuations de température de moins de $1^{\circ}$; puis un échantillon montre une phosphatase de 0,17 . Le crémage est extrêmement rapide : $t_{1 \%}=0,1$. Conclusion : température trop basse. L'inspection fait découvrir que l'exploitation elle-même trouvait aussi de la phosphatase, mais le diagramme de température était exact. L'inspecteur trouve le motif : le thermomètre, détaché à la suite de tremblement, indiquait 104 trop haut.

Une nouvelle exploitation installee à l'extérieur d'Amsterdam livre son premier échantillon. La réaction de Storch est positive. La vitesse de crémage est pareille à celle du lait cru. La pasteurisation est donc basse ou très basse. Il y a une teneur en phosphatase petite, mais visible. Conclusion : température de pasteurisation un peu trop basse, ou bien infiltration de lait cru. Après discussion, on élève de peu la température, et la teneur en phosphatase disparaît. La faute commise n'était probablement que la trop basse température.

La facilité avec laquelle des déviations naissent, si le travail est moins consciencieux, apparaît lors de l'examen de lait pasteurisé vendu libre (" non embouteillé ») et qui n'a pas été entouré d'autant de soins. Du lait écrémé, qui, dans ce cas, par contrat, devait être livré avec réaction négative de Storch, donne à plusieurs reprises des chiffres de phosphatase de 0,10 a 0,20 et Storeh \pm ou (Dans ce cas, la peroxydase fut déterminée quantitavement aussi). Conclusion : mélange de lait cru ou insuffisamment chauffé à une masse principale chauffée à température élevée. Inspection : la pasteurisation s'effectue au moyen d'un type ancien d'appareil à écoulement, où le lait est mal mélangé pendant son passage, et où toutes les parties du liquide ne viennent pas à la température désirée (tableau $\mathrm{G}, \mathrm{n}^{\mathrm{0s}} 9$ et 10 ).

Du lait de restaurant est examiné sur place par l'inspecteur au moyen de la réaction de "Storch». Dans le cas d'un résultat + (défendu par le règlement communal), on prélève des échantillons et on détermine la phosphatase. Parfois, le résultat est négatif : on a donc employé du lait sous-pasteurisé. Parfois aussi, nous trouvons : phosphatase $=0,15-0,40$ environ.

Dans ce cas, on peut admettre que le tenancier du restaurant a ajouté du lait eru (emploi de la même vaisselle pour le lait cru). En de pareils cas, le défaut a toujours disparu après avertissement, ainsi qu'un examen renouvelé l'a montré*.

(*) Le décret Staatscourant 74, du 9 avril 1941, exige en Hollande, pour tout lait pasteurisé et vendu en bouteille, que la réaction phosphatase soit négative, et qu'en même temps, des précautions soient prises dans la production, ce qui auparavant n'était preserit que pour la ville d'Amsterdam. 